\title{
Revisiting the effect of red on competition in humans
}

\author{
Laura Fortunato ${ }^{1,2, *}$ and Aaron Clauset ${ }^{3,4,2, \dagger}$ \\ ${ }^{1}$ Institute of Cognitive and Evolutionary Anthropology, University of Oxford, Oxford OX2 6PN, UK \\ ${ }^{2}$ Santa Fe Institute, Santa Fe, NM 87501, USA \\ ${ }^{3}$ Department of Computer Science, University of Colorado, Boulder, CO 80309, USA \\ ${ }^{4}$ Biofrontiers Institute, University of Colorado, Boulder, CO 80303, USA
}

7

Bright red coloration is a signal of male coms petitive ability in animal species across a range 9 of taxa, including non-human primates. Does the 10 effect of red on competition extend to humans? A 11 landmark study in evolutionary psychology estab12 lished such an effect through analysis of data for ${ }_{13}$ four combat sports at the 2004 Athens Olympics ${ }_{14}[1]$. Here we show that the observed pattern re15 flects instead a structural bias towards wins by 16 red in the outcomes of the competition. Consis17 tently, we find no effect of red in equivalent data ${ }_{18}$ for the 2008 Beijing Olympics, which present a 19 structural bias towards wins by blue. These re20 sults refute past claims of an effect of red on hu21 man competition based on analysis of this sys22 tem. In turn, this undermines the notion that ${ }_{23}$ any effect of red on human behavior is an evolved response shaped by sexual selection.

24

25 In animal species across a range of taxa, bright col${ }_{26}$ oration is a secondary sexual character acting as a signal ${ }_{27}$ of male competitive ability [2]. In mandrills, for example, 28 male rank is determined through contest competition, 29 with marked reproductive skew in favor of top-ranking 30 individuals. High rank is associated with better repro31 ductive outcomes also in females, but here rank is in32 herited from the mother instead. As expected within the 33 framework of Darwinian sexual selection [3, 4], the extent 34 and intensity of red skin on the face of adult individuals 35 vary with rank in males, but not in females $[2,5]$.

The relationship between red coloration and compe37 tition in non-human primates and other taxa raises an 38 intriguing question [1]: does red have an effect on the 39 outcome of human competitive interactions, shaped by 40 similar evolutionary processes? Of course, humans do not 41 present natural displays of conspicuous secondary sexual 42 coloration. However, increased or decreased blood flow 43 to the skin are linked to a range of emotional states, in${ }_{44}$ cluding anger and fear. This response may serve as a 45 subtle cue of relative dominance during aggressive en46 counters, echoing the sexually selected response to red in 47 other species.

48 Hill \& Barton [1] reasoned that the effect may ex49 tend to artificial stimuli, for example wearing red dur50 ing a physical contest. In an ingenious first test of this

\footnotetext{
* laura.fortunato@anthro.ox.ac.uk

$\dagger$ aaron.clauset@colorado.edu
}

51 hypothesis, they exploited a structural feature of tour52 naments in four Olympic combat sports: boxing, taek53 wondo, Greco-Roman wrestling, and free-style wrestling. ${ }_{54}$ In these sports, contestants compete in pairs as red vs. 55 blue, with distinctively colored clothing and/or equip56 ment. In the 2004 Olympics, colors were assigned to 57 contestants independent of ability. If red does confer ${ }_{58}$ a competitive advantage, as predicted, then contestants 59 wearing red would be more likely to defeat their oppo60 nents, and more than half the contests would end in a 61 win by red.

62 Data on outcomes in the men's divisions for the four ${ }_{63}$ sports at the 2004 Athens Olympics upheld this predic${ }_{64}$ tion [1] (Fig. 1a), and no effect was found in the two 65 sports with women's divisions (taekwondo and free-style ${ }_{66}$ wrestling) [6]. These patterns were taken to support the 67 hypothesis of a red advantage in human competitive in68 teractions: red enhances performance, possibly acting as 69 a cue of relative dominance when factors such as skill or 70 strength are equally matched. At the proximate level, 71 the effect was posited to operate through psychological 72 or physiological (e.g., hormonal) influences on the red73 wearing competitor, on his opponent, or both [6].

We present an alternative explanation, which fully ac75 counts for the observed pattern without recourse to an 76 effect of red on competitive outcomes. In the four sports 77 analysed, the competition for a given weight class is 78 arranged as a single-elimination tournament (Fig. 1b). ${ }_{79}$ While details vary across sports (Supplementary Infor80 mation), generally the winner of a contest, or bout, pro81 ceeds to the next round in the competition "tree". In 82 boxing and wrestling, the contestant placed at the top of 83 the bout wears red, the one placed at the bottom wears ${ }_{84}$ blue; the pattern is reversed in taekwondo. A contes85 tant's relative position, and thus the color he wears, may ${ }_{86}$ change between bouts, as he progresses through rounds 87 in the tournament (Fig. 1b).

${ }_{88}$ When the tournament structure is incomplete and con89 testants vary in skill level, the null distribution for the 90 fraction of red wins can depart from 0.5, due to a bias ${ }_{91}$ towards wins by one color in the outcomes of the com92 petition (Supplementary Information). Two sources of 93 incompleteness are byes and walkovers, both of which 94 result in "missing" bouts (Fig. 1b; Supplementary In95 formation). Using a Monte Carlo simulation of compe96 tition [7] on the actual 2004 tournament structures, we 97 numerically calculated the distribution of red wins under 98 the null hypothesis (no effect of red), for different degrees 99 of variance in competitor skill (Methods). Compared to 
a

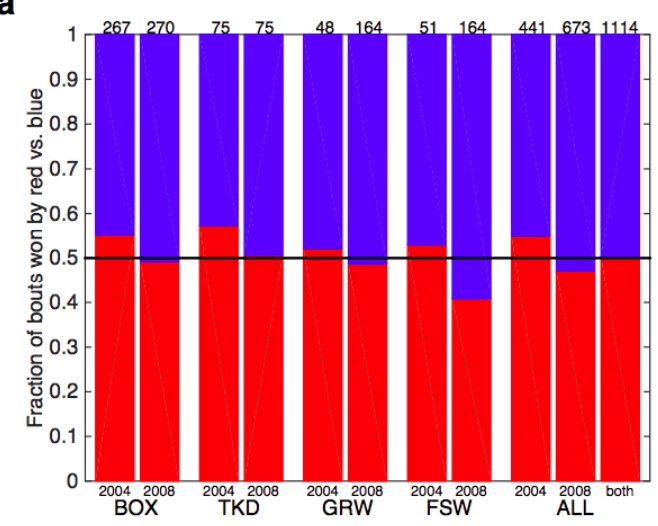

C

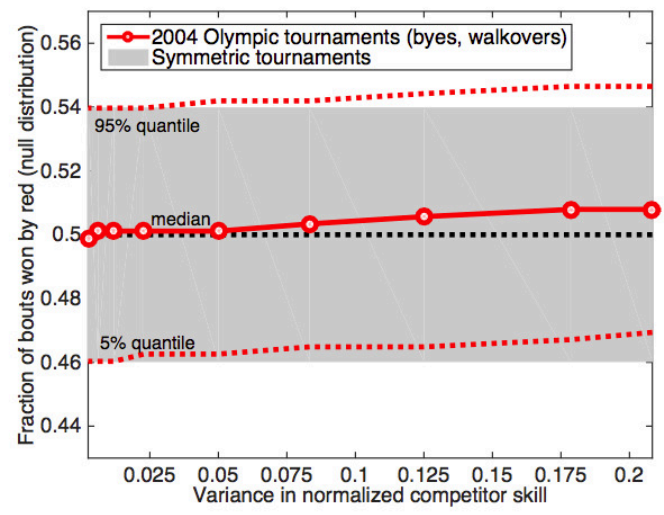

b

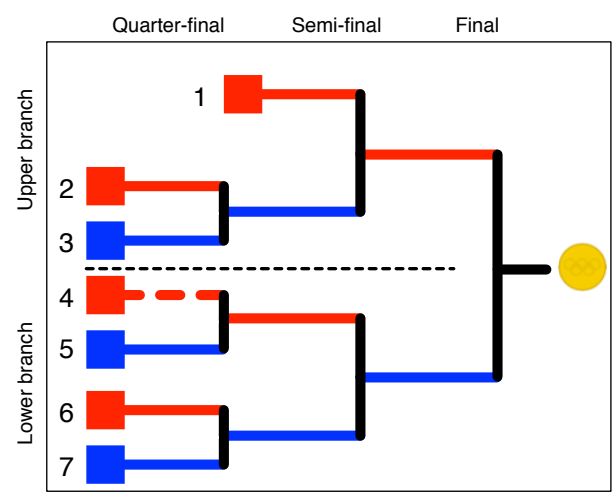

\section{d}

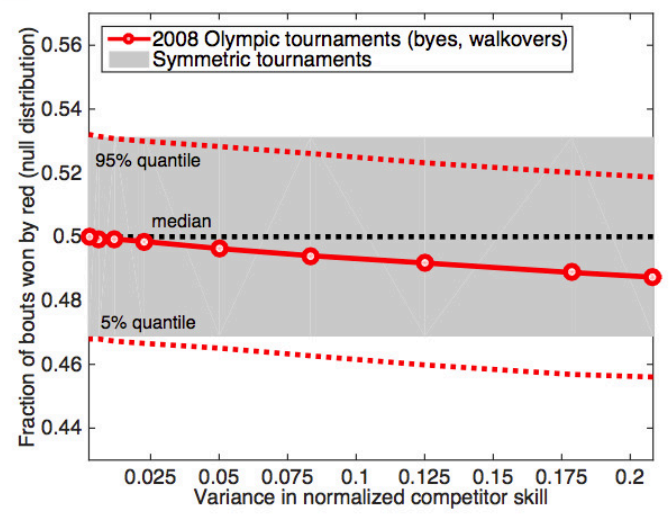

FIG. 1. Testing the effect of red in Olympic contests. a, Fractions of bouts won by contestants wearing red vs. blue in the male divisions of boxing (BOX), taekwondo (TKD), Greco-Roman wrestling (GRW), and free-style wrestling (FSW) at the 2004 Athens and 2008 Beijing Olympics, along with fractions when outcomes are aggregated over the four sports (ALL) by year (2004, 2008) or over the two years (both). The number of bouts in each group is reported above the corresponding bar. The horizontal line shows $f_{\text {red }}=0.5$. See Table I for details. b, Schematic representation of the structure of a single-elimination tournament for $n=7$ contestants. Because $n$ is not a power of 2 , the outermost round is incomplete. In this case, contestant 1 does not compete in the quarter-final round, i.e., he is byed to the semi-final round. In each contest, or bout, the contestant at the top wears red, the one at the bottom wears blue. For example, contestants 2 and 3 wear red and blue, respectively, in the quarter-final round. The winner of this bout proceeds to the semi-final round (in blue), where he faces contestant 1 (in red). The bout between contestants 4 and 5 is won by walkover (dotted red line, indicating that contestant 4 withdrew or failed to show up). Contestant 5 proceeds to the semi-final round (in red), where he faces the winner of the 6-7 bout (in blue). c,d, Quantiles for the distribution of the fraction of red wins $f_{\text {red }}$ under the null hypothesis on the actual asymmetric tournament structures in the 2004 Athens and the 2008 Beijing Olympics (red line) and an equivalently sized symmetric tournament (grey fill). In both cases, the distributions were evaluated by Monte Carlo at the locations of the red dots. Asymmetries in the tournament structures shift the null distribution away from a mean of $f_{\text {red }}=0.5$ as skill variance increases. These asymmetries induced a bias towards red in 2004 and towards blue in 2008. See text for details.

100 equivalent tournaments with no missing bouts, the null 111 This interpretation is further supported by equivalent 101 distribution for the incomplete tournaments shifts in fa- 112 data for the 2008 Olympics (Supplementary Informa102 vor of red wins as skill variance increases (Fig. 1c). This 113 tion). We find no evidence of a red effect (Fig. 1a and Ta${ }_{103}$ implies that a standard hypothesis test will overstate the $114 \mathrm{ble}$ I), and Monte Carlo simulations show that in this case ${ }_{104}$ statistical significance of any observed pattern favoring 115 the pattern of incompleteness induces a bias towards wins 105 red (Supplementary Information), and a correctly param- 116 by blue in the outcomes of the competition (Fig. 1d). 106 eterized test of the red hypothesis cannot be constructed ${ }_{117}$ Furthermore, data pooled over both years show no ev107 without knowing the true variance in skill. A conserva- 118 idence of a red effect (Fig. 1a and Table I). Finally, an ${ }_{108}$ tive interpretation, however, is that the pattern reported 119 estimate of the statistical power indicates that if an effect 109 by Hill \& Barton [1] reflects this underlying bias in the ${ }_{120}$ does indeed exist in these data, it must be small, altering 110 null distribution (Table I; Supplementary Information). ${ }_{121}$ the outcome in no more than $1.3 \%$ of bouts relative to 


\begin{tabular}{|c|c|c|c|c|c|c|}
\hline Year & Test & Sport (s) & $n_{\text {red }}$ & $n$ & $f_{\mathrm{red}}$ & $p$-value \\
\hline \multirow{7}{*}{ 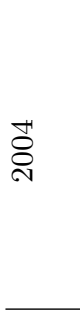 } & Bouts & $\mathrm{BOX}$ & 147 & 267 & 0.551 & 0.056 \\
\hline & Bouts & TKD & 43 & 75 & 0.573 & 0.124 \\
\hline & Bouts & GRW & 25 & 48 & 0.521 & 0.443 \\
\hline & Bouts & FSW & 27 & 51 & 0.529 & 0.390 \\
\hline & Bouts & ALL & 242 & 441 & 0.549 & 0.023 \\
\hline & Rounds & ALL & 16 & 21 & 0.762 & 0.013 \\
\hline & Weight classes & ALL & 19 & 29 & 0.655 & 0.068 \\
\hline \multirow{7}{*}{$\stackrel{\infty}{\stackrel{\sim}{\circ}}$} & Bouts & $\mathrm{BOX}$ & 133 & 270 & 0.493 & 0.620 \\
\hline & Bouts & TKD & 38 & 75 & 0.507 & 0.500 \\
\hline & Bouts & GRW & 80 & 164 & 0.488 & 0.652 \\
\hline & Bouts & FSW & 67 & 164 & 0.409 & 0.992 \\
\hline & Bouts & ALL & 318 & 673 & 0.473 & 0.929 \\
\hline & Rounds & ALL & 8 & 25 & 0.320 & 0.978 \\
\hline & Weight classes & ALL & 11 & 29 & 0.379 & 0.932 \\
\hline Both & Bouts & ALL & 560 & 1114 & 0.503 & 0.440 \\
\hline
\end{tabular}

TABLE I. Tests of a red effect in Olympic contests. Results for tests of a red effect in the male divisions of boxing (BOX), taekwondo (TKD), Greco-Roman wrestling (GRW), free-style wrestling (FSW), and aggregated over the four sports (ALL), at the 2004 Athens and 2008 Beijing Olympics. Tests denoted "bouts" compare the number of bouts won by red, $n_{\text {red }}$, to the $n$ total wins. Tests denoted "rounds" compare the number of rounds with a majority of red wins, $n_{\text {red }}$, to the $n$ total rounds. Tests denoted "weight classes" compare the number of weight classes with a majority of red wins, $n_{\text {red }}$, to the $n$ total weight classes. In all cases, $f_{\text {red }}=n_{\text {red }} / n$. Reported are the results of one-sided binomial tests $\left(H_{0}: f_{\text {red }} \leq 0.5 ; H_{\mathrm{A}}: f_{\text {red }}>0.5\right)$, with $\alpha=0.05$. None of the results are significant under a Bonferroni-adjusted threshold $\alpha_{\mathrm{c}}=0.003$ (Supplementary Information). 122 natural variation. In fact, this value likely overestimates 159 progressively over rounds. When a pair of competitors $r$ ${ }_{123}$ the true impact, as it is calculated without accounting 160 and $b$ faced off, $r$ advanced to the next round with prob124 for the structural biases described above (Supplementary ${ }_{161}$ ability $x_{r} /\left(x_{r}+x_{b}\right)$ [7] (Supplementary Information).
${ }_{125}$ Information).

126 These findings suggest that red does not affect the out127 comes of Olympic contests, challenging past claims about 128 the role of color in human competitive interactions based 129 on analysis of this system $[1,6]$. Moreover, our analy130 sis illustrates that confounding effects arising from non131 independence and biases in the data-generating process, 132 multiple hypothesis testing, and low statistical power can 133 be subtle (Supplementary Information). Extreme cau134 tion is thus required in interpreting related results de135 rived from other systems [reviewed in 8,9].

A large body of work has developed over the past ${ }_{137}$ decade, building on the hypothesis of a sexually selected 138 response to red in humans [reviewed in 8,9] — indeed, 139 the effect of red on human behavior has come to be re140 garded as one of the best established, and most salient, ${ }_{141}$ in the field of color psychology, with important practi142 cal applications [10]. Our results refute the foundational ${ }_{143}$ finding to this body of work [1], casting doubt on claims 144 that any effect of red on human competition has an evo145 lutionary basis. In what way evolution has shaped the 146 human response to color, and how this is reflected in ${ }_{147}$ present-day human behavior, remain open questions.

\section{${ }_{48}$ Methods}

149 Details of the data collection and analysis are in the Sup150 plementary Information. Null distributions for $f_{\text {red }}$ were 151 obtained by Monte Carlo simulation of single-elimination 152 tournaments, by weight class, sport, and year. Results 153 were then aggregated for analysis. Each simulated weight 154 class used its observed tournament structure, including 155 asymmetries (byes, walkovers). Competitors were as156 signed randomly to initial tournament positions, with ${ }_{157}$ skill levels drawn i.i.d. from a symmetric Beta distribu158 tion: $x \sim \operatorname{Beta}(\beta, \beta)$. Bout outcomes were evaluated
[1] Hill, R. A. \& Barton, R. A. 2005 Red enhances human 179 performance in contests. Nature 435, 293-293.

[2] Setchell, J. M. 2015 Color in competition contexts in non- 181 human animals. In Handbook of color psychology (edited 182 by A. J. Elliot, M. D. Fairchild, \& A. Franklin), chap. 26, 183 pp. 546-567. Cambridge: Cambridge University Press. ${ }_{184}$

[3] Darwin, C. 1871 The descent of man, and selection in 185 relation to sex. London: John Murray.

[4] Darwin, C. 1876 Sexual selection in relation to monkeys. ${ }_{187}$ Nature 15, 18-19.

[5] Setchell, J. M. 2016 Sexual selection and the differ- 189 ences between the sexes in mandrills (Mandrillus sphinx). 190 American Journal of Physical Anthropology 159, 105- 191 129.

[6] Barton, R. A. \& Hill, R. A. 2005 Sporting contests: See- 193 ing red? Putting sportswear in context (reply). Nature 437, E10-E11.
[7] Bradley, R. \& Terry, M. 1952 Rank analysis of incomplete block designs. i. The method of paired comparisons. Biometrika 39, 324-345.

[8] Wiedemann, D., Barton, R. A., \& Hill, R. A. 2012 Evolutionary perspectives on sport and competition. In $A p$ plied evolutionary psychology (edited by S. C. Roberts), chap. 18, pp. 290-307. Oxford: Oxford University Press.

[9] Maier, M. A., Hill, R. A., Elliot, A. J., \& Barton, R. A. 2015 Color in achievement contexts in humans. In Handbook of color psychology (edited by A. J. Elliot, M. D. Fairchild, \& A. Franklin), chap. 27, pp. 568-584. Cambridge: Cambridge University Press.

[10] Elliot, A. J. \& Maier, M. A. 2014 Color psychology: effects of perceiving color on psychological functioning in humans. Annual Review of Psychology 65, 95-120. 